\title{
The Effect of National Weekly COVID-19 Screening Testing of All Workers in Long-Term Care Facilities: a Decrease in Mortality
}

Nimrod Maimon ( $\square$ NIMROD.MAIMON@MOH.GOV.IL)

Ministry of Health Israel

Michal Maimon

Soroka University Medical Center

Lior Hassan

Soroka University Medical Center

Itamar Grotto

Ministry of Health

Yasmeen Abu-Fraiha

Soroka University Medical Center

Shahar Robinson-Geva

Soroka University Medical Center

Limor Gortzak Uzan

Ministry of Health

Sivam Goldin

Ministry of Health

Victor Novack

Soroka University Medical Center

\section{Article}

Keywords: COVID-19, testing, screening, long-term care facilities

Posted Date: February 2nd, 2021

DOl: https://doi.org/10.21203/rs.3.rs-153758/v1

License: (c) (i) This work is licensed under a Creative Commons Attribution 4.0 International License.

Read Full License 


\section{Abstract}

Background Outbreaks of Coronavirus 2019 (COVID-19) in Long-Term Care Facilities (LTCFs) have resulted mainly from disease transmission by asymptomatic health care workers (HCW's). It is not known whether routine COVID-19 screening tests carried out on HCW's would reduce mortality of LTCF residents. Since mid-July 2020, the Israeli national LTCF defense program - "Senior Shield" - has used weekly COVID-19 PCR tests on all LTCF employees.

Methods A nationwide, government funded, screening program on all LTCF personnel for four months during the second COVID-19 wave. We evaluated differences between the two waves in the national LTCF's system with regard to hospitalizations and mortality. Estimation of national health system predicted outcomes, in the absence of this weekly screening plan, was calculated.

Results COVID-19 tests were taken weekly in all 1,107 LTCFs which includes 62,159 HCWs and 100,046 residents. A median of 55,282 (range - 16,249) tests were performed each week. Turnaround time from sampling to result was less than 24 hours in $95 \%$ of tests. Compared to the first wave, in which $45.3 \%$ of national mortality was attributed to the LTCF's, the second wave saw a $33.8 \%$ reduction in this mortality ratio. Estimation of national health system outcomes during the second wave showed that the activation of the screening program reduced hospital load by $35 \%$ and prevented $30 \%$ of national mortality from COVID-19.

Conclusions Routine weekly COVID-19 PCR testing of all LTCF employees may reduce national hospitalizations and mortality.

\section{Background}

COVID-19 outbreaks and resident deaths in LTCFs account for $30-80 \%$ of all COVID-19-related mortality in many western countries and these catastrophic events have driven up the total case-fatality rate worldwide [1-4]. LTCF residents are both elderly and have multiple comorbidities with a high prevalence of cognitive impairment, making it challenging to appropriately apply barriers to infection. Moreover, most LTCFs are overcrowded and are poorly prepared to implement infection control policies owing to a shortage of staff, personal protective equipment and the relatively low level of HCW training [5-8]. These factors make LTCFs extremely vulnerable to deadly COVID-19 outbreaks [9-10].

During the first wave of the epidemic, Israel, which is, in effect, almost completely isolated from surrounding countries, reacted with the prompt closure of the skies and an early lockdown. This quick response resulted in relatively low COVID-19 morbidity in the first wave with a maximum weekly average incidence of 5.4/100,000 and a low mortality rate of 30.9/1,000,000 (compared to 95/100,000 and $110 / 1,000,000$ respectively in EU countries). Nevertheless, LTCF mortality at the peak of the wave accounted for $52.4 \%$ of total national deaths [11-12]. Consequently, public pressure, as well as expert opinion led the government to establish a dedicated national level task force with the aim of protecting the LTCF sector from the spread of COVID-19, in mid-April 2020. This task force, "Senior Shield", ("Magen 
Avot v'Imahot") is a government agency tasked with supporting the efforts of the ministerial organizations and LTCFs in managing the COVID-19 crisis, and shielded all LTCFs throughout the country [13]. A too-rapid return to normal life led to substantial spread of COVID-19 and a huge second wave of infection, starting in August 2020, reached a peak in September-October 2020 with a weekly average incidence of $800 / 100,000$ new positive cases and 15\% of COVID-19 PCR tests being positive.

In order to reduce morbidity and mortality in LTCFs, and to lower the burden on public health systems, the Centers for Disease Control and Prevention (CDC) and European Centre for Disease Prevention and Control (ECDC) Guidance recommended routine testing of all HCWs and active facility surveillance if there is a confirmed case of COVID-19 $[14,15]$. Those recommendations were in concordance with our observation that $91 \%$ outbreaks in LTCFs, in the first wave had been caused by asymptomatic HCWs. Therefore, we took the CDC and ECDC testing strategies a step further, and starting in mid-July 2020 the

"Senior Shield" national task force executed a routine weekly PCR testing and screening program for COVID-19 infection of all LTCF workers. This report presents the results of this nationwide program to reduce the burden of COVID-19 on the LTCF sector.

\section{Methods}

\section{Study Design and Participants.}

This study included all residents of, and HCWs in, Israeli LTCFs who were screened weekly during the second wave of COVID-19 which occurred between July 132020 and November 21 2020. We compared morbidity and mortality of COVID-19 on LTCF residents before and after the implementation of a weekly, nationwide, government-funded, PCR screening of LTCF personnel for COVID-19.

LTCFs across Israel encompass a broad range of types of institutions ranging from home-like facilities to those providing specialized medical care. These facilities include long-term care hospitals, skilled nursing facilities, nursing homes, residential homes, hostels for people with disabilities, palliative care and rehabilitation centers. The wards are categorized on the basis of the physical and cognitive function of their residents: independent, semi-independent, cognitively frail, frail, requiring simple nursing care and requiring skilled nursing care.

\section{Definitions}

We defined an "outbreak event" as being where at least one resident in a LTCF was positive for COVID-19, an "avoided outbreak event" as being where a single employee of a LTCF was discovered to be positive in the screening test but, in the subsequent 2 weeks of comprehensive LTCF testing, no resident was infected. "Decreased size outbreak event" was defined as being where a maximum of 5 residents were infected in the two weeks following a COVID-19 positive result in a facility employee by the screening program.

\section{Procedures}


Each LTCF in Israel was screened by the Israeli Emergency Medical Services ("Magen David Adom") on a preset weekday. The specimens were transferred directly to a single PCR lab ("My Heritage") that was dedicated to this project. The test results were reported directly to the LTCF medical directors and also to "Senior Shield" headquarters within 24 hours. When an employee was identified as positive for COVID-19, an outbreak response plan was activated immediately by the "Senior Shield" headquarters in collaboration with the National Infection Control Team, under the local supervision of the Ministry of Health public health and the Ministry district geriatrician. The employee was isolated and all of his colleagues with whom he had been in contact, as well as exposed LTCF residents, were screened within 24 hours and subsequently every 3 days in order to detect other asymptomatic carriers. This screening was continued until all employees and residents were negative for three consecutive tests (Fig. 1). Asymptomatic residents were isolated in special COVID-19 wards within the LTCF or by transfer to dedicated COVID-19 wards in another geriatric facility. Symptomatic residents were admitted to a COVID19 ward in general hospitals.

\section{Statistics}

\section{Database}

We collected data on LTCF residents and HCWs who were diagnosed during the period of the first wave of COVID-19, between March 212020 and July 13 2020, and to the end of the second wave at November 21, 2020. The data were aggregated to a weekly sum of the number of COVID-19 PCR tests, infected residents and HCWs, and resident mortality and hospitalization. The Israeli COVID-19 demographic statistics were taken from the Ministry of Health COVID-19 database during the periods mentioned.

\section{Statistical analysis}

We compared the proportion of positive COVID-19 PCR tests, hospitalizations, overall national mortality, and mortality in LTCF residents over age 75 , before and during the implementation of the national program. Variables are described with numbers and percentages. In addition, comparisons between the two time periods are presented by $p$-values. Percentages are rounded to one decimal place. Study variables were tested using Pearson's $\chi 2$ test for contingency tables or Fisher Exact test, as appropriate.

Statistical analysis was performed using R-studio (RStudio Team (2020). Rstudio: Integrated Development for R. Rstudio, PBC, Boston, MA URL http://www.rstudio.com/.) and python 3.9 (Python Software Foundation. Python Language Reference).

The study was determined to be exempt by the Institutional Review Board of Soroka University Medical Center and thus informed consent was not required (0429-20-SOR). This study followed the Strengthening the Reporting of Observational Studies in Epidemiology (STROBE) reporting guideline.

\section{Results}

The program incorporated all 1,107 of the relevant licensed institutions in the country, involving 100,046 residents (1.1\% of the Israeli population), as well as 62,159 employees (Table 1). One hundred percent of 
LTCFs joined the program and the weekly average compliance of HCWs was $88.5 \%$. The program screened a median of 55,282 (Range - 16,249) employees per week. The employee screening tests were positive in $0.05-1.5 \%$ of cases and the weekly percentage of positive screening tests was similar to the incidence of COVID-19 in the general population during the second wave. During the 19 week screening program 2,673 asymptomatic HCWs and 3,505 residents were found to be positive and were isolated.

\section{Mortality}

We compared COVID-19 mortality in LTCFs as a percentage of national COVID-19 mortality in both the first and second waves of the COVID-19 epidemic (Fig. 2). We found that in the first wave deaths from COVID-19 in LTCFs accounted for 45.3\% (252/556) of all COVID-19 deaths recorded nationally, and in the second wave this ratio was reduced to $30.3 \%(709 / 2,337)$ representing a reduction of $33.8 \%$ in expected mortality $(\mathrm{P}<0.01)$. Analyzing only the peaks of the waves - LTCF deaths, as a proportion of total national deaths, reached $51.2 \%(60 / 117)$ at the crest of the first wave compared to $31.4 \%(233 / 742)$, at the crest of the second wave which signifies a reduction of $38.6 \%$ in expected mortality $(P<0.01)$. Additionally, Fig. 2 demonstrates that this decrease started two weeks after the implementation of the screening program in mid-July 2020.

\section{Outbreaks, decreased sized outbreaks, and avoided outbreaks}

During the screening period we had 912 outbreak events compared to only 235 outbreaks in the first wave. A decreased size outbreak means that we were able to contain the event and limit the number of positive patients. In the first wave $20.4 \%(48 / 235)$ could be defined as decreased sized outbreaks, compared to $82.8 \%$ (755/912) in the second wave. Furthermore, there were 214 cases of avoided outbreaks in which the early identification of COVID-19 positive HCWs prevented subsequent infections in the facility.

\section{Discussion}

We describe the results of the national program of periodic universal staff screening of all of Israel's LTCFs. Our analysis indicates that implementation of such a weekly screening program for LTCF employees resulted in a substantial reduction in the mortality ratio in the second wave compared to the first wave.

During the COVID-19 pandemic, LTCFs have been a major source of devastating outbreaks in many countries accompanied by high mortality and morbidity, causing a consequential load on national health systems $[1,2]$. Root-cause analysis of LTCF outbreaks identified that COVID-19 prevalence in the community is the main factor that is directly associated with the likelihood of COVID-19 entering the facility [11, 18-22]. Accordingly, this program involves primary prevention of outbreak events by identifying asymptomatic HCWs and potentially preventing the outbreak entirely. Additionally, it includes a second line of defense, immediately activating the screening of all residents and employees, where a positive COVID-19 case is identified, enabling us to limit the extent of the outbreak. The PCR test results were reported directly to the LTCF medical directors, within 24 hours, and the response was immediately 
implemented out by the Senior Shield program headquarters, which stood by ready to take action on a 24/7 basis [23].

Evaluation of international ability to defend the LTCF sector from COVID-19 mortality is a challenging task due to differences in the number of LTCF beds per population. Israel's population is significantly younger than in other western countries (Israel has only $11.2 \%$ above 65 compared to OECD $18.3 \%$ and USA 16.5\%) and the number of LTCF residents per million is lower than most OECD countries [15, 24]. An ECDC report showed that general mortality in the LTCF sector seems to be directly correlated with the prevalence of COVID-19 in the country. However, according to this report, even though Israel had a high prevalence of COVID-19 cases, there was very low LTCF mortality per 1000 beds (Fig. 3). Given that health system accessibility as well as medical practice to COVID-19 patients during the course of the pandemic was identical across Israel, the decrease in the mortality ratio of LTCF residents in the second wave, compared to the first wave, can be accounted for by the execution of the LTCF screening plan.

During the COVID-19 pandemic the Ministry of Health defined the national "red line capacity" level of Israel hospital system to be no more than 800 patients hospitalized with severe COVID-19. During the peak of the second wave Israel crossed this red line and had 898 such patients. The activation of this program, with the reduction in LTCF outbreak events and the associated decrease in morbidity and acute hospitalizations, significantly contributed to preventing the national public hospital system from being overwhelmed during the second wave (Table 2). Our experience with this screening program indicated an ability to offer a sustained reduction in overall outbreaks up to a certain level of community spread of COVID-19. However, when the test positivity rate increased above $5 \%$, and above a weekly incidence of $240 / 1,000,000$ in the Israeli population, in the second wave, a bi-weekly screening became mandatory in order to prevent outbreaks and to better defend these vulnerable residents.

Before starting the program there were three main concerns: first that HCWs with negative COVID-19 test results would weaken their compliance with the use of face masks and would not follow mandatory hygiene procedures. Second, the ability to oblige LTCFs to join the program and the HCWs to undergo repeated weekly testing was weak. Interestingly we noticed that the staff's acceptance of screening as an effective way of protecting the LTCF's residents, resolved this issue rapidly. This is demonstrated by the $100 \%$ compliance of facilities in joining the program and the adherence of managers to screening more than $90 \%$ of their workers every week. Third, given Israel's strict medical confidentiality laws there were legal obstacles to transferring the employee's medical information (COVID-19 test results) to their direct administrative manager. We were able to resolve this issue by permitting the results to be transferred to the LTCF's medical director.

This report has some limitations. Bear in mind that this was and continues to be a real-life pandemic situation with all of the confusion and uncertainty engendered by such crises. As such it took one month from initiating the system to reaching full coverage of all LTCFs. Therefore, the results are not as precise as they should be in a well-designed study. However, the dramatic differences in LTCF resident mortality 
and morbidity in the two waves, which started two weeks after the implementation of the screening program and continued during the second wave - strengthens our impression that such a national or local screening program is effective in reducing morbidity and mortality in this especially vulnerable group of older persons.

In this study we found that by using weekly PCR testing of all employees we were able to protect the LTCF system from outbreaks. Although immunization of LTCF residents and HCW is the ultimate goal in preventing further COVID-19 infection, our research suggests an effective method of protecting LTCF against future outbreaks caused by seasonal respiratory pathogens. We conclude that adopting a program of routine employee screening may reduce LTCF resident mortality and morbidity, and may help prevent national health systems from being overwhelmed.

\section{References}

1. Long Term Care Response to COVID-19 International Long Term Policy Network https://ltccovid.org/country-reports-on-covid-19-and-long-term-care/

2. DATA.CMS.GOV. COVID-19 Nursing Home Data. https://data.cms.gov/stories/s/COVID-19-NursingHome-Data/bkwz-xpvg/

3. Coronavirus (COVID-19) current situation and case numbers: Coronavirus COVID-19) at a glance. https://www.health.gov.au/sites/ default/files/documents/2020/09/coronavirus-covid-19-ataglance-14-september-2020.pdf Australian Government Department of Health.

4. Canadian institute of health Information Pandemic experience in the long-term care sector: How does Canada compare with other Countries? 2020.

5. Arons MM, Hatfield KM, Reddy SC. Presymptomatic SARS-CoV-2 infections and transmission in a skilled nursing facility. N Engl J Med. 2020; 382:2081-2090.

6. Gwendolyn L. Gilbert. COVID-19 in a Sydney nursing home: a case study and lessons learnt. Med J Aust. 2020 Nov; 213(9): 393-396.e1

7. Grabowski DC, Mor V. Nursing Home Care in Crisis in the Wake of COVID-19. JAMA. 2020; 324 (1):23-24. doi:10.1001/jama.2020.

8. Niu S, Tian S, Lou J. Clinical characteristics of older patients infected with COVID-19: A descriptive study. Arch Gerontol Geriatr. 2020; 89: 104058.

9. McMichael TM, Currie DW, Clark S. Epidemiology of Covid-19 in a Long-Term Care Facility in King County, Washington. N Engl J Med. 2020; 382: 2005-2011.

10. Abrams HR, Loomer L, Gandhi A, Grabowski DC. Characteristics of U.S. Nursing Homes with COVID19 Cases. J Am Geriatr Soc. 2020 Aug;68(8):1653-1656. doi: 10.1111/jgs.16661. Epub 2020 Jul 7. PMID: 32484912; PMCID: PMC7300642.

11. European Centre for Disease Prevention and Control, An agency of the European Union. https://www.ecdc.europa.eu/en/covid-19-pandemic 
12. Israeli Ministry of Health - COVID - 19 Dashboard. https:// datadashboard. health.gov.il/ COVID 19 / general? utm_source = go. gov. il \& utm_medium = referral

13. "Senior Shield" - The Israeli National COVID-19 Protection Program for Long Term Care Facilities. https://govextra.gov.il/ministry-of-health/care-covid19/elderly-care-covid19/

14. CDC. Coronavirus disease 2019 (COVID-19): preparing for COVID-19 in nursing homes. Atlanta, GA: US Department of Health and Human Services, CDC; 2020. https://www.cdc.gov/coronavirus/2019ncov/hcp/long-term-care.html.

15. European Centre for Disease Prevention and Control. Increase in fatal cases of COVID-19 among long-term care facility residents in the EU/EEA and the UK. 19 November 2020. ECDC: Stockholm; 2020.

16. Zhang J, Litvinova M, Liang Y, et al. Changes in contact patterns shape the dynamics of the COVID19 outbreak in China. Science. Jun 2020: Vol. 368, Issue 6498, pp. 1481-1486. DOI: 10.1126/ science. abb8001

17. Zou L, Ruan F, Huang M. SARS-CoV-2 viral load in upper respiratory specimens of infected patients. N Engl J Med. 2020; 382:1177-1179.

18. Justin Blackburn, Lindsay Weaver, Liza Cohen, et al. Community Coronavirus Disease 2019 Activity Level and Nursing Home Staff Testing for Active Severe Acute Respiratory Syndrome Coronavirus 2 Infection in Indiana. Journal of the American Medical Directors Association. 2020. ISSN 1525-8610, https://doi.org/10.1016/j.jamda. 2020.10.038.

19. Brown KA, Jones A, Daneman N, et al. Association Between Nursing Home Crowding and COVID-19 Infection and Mortality in Ontario, Canada. JAMA Intern Med. 2020 Nov 9:e206466. doi:10.1001/jamainternmed. 2020.6466. Epub ahead of print. PMID: 33165560; PMCID: PMC7653540.

20. Jennifer K Burton, Gwen Bayne, Christine Evans, et al. Evolution and effects of COVID-19 outbreaks in care homes: a population analysis in 189 care homes in one geographical region of the UK.

21. Gorges RJ. and Konetzka RT. Staffing Levels and COVID-19 Cases and Outbreaks in U.S. Nursing Homes. J Am Geriatr Soc, 2020, 68: 2462-2466. https://doi.org/10.1111/jgs.16787

22. He M, Li Y, Fang F. Is There a Link between Nursing Home Reported Quality and COVID-19 Cases? Evidence from California Skilled Nursing Facilities. J Am Med Dir Assoc. 2020 Jul;21(7):905-908. doi: 10.1016/j.jamda.2020.06.016. Epub 2020 Jun 15. PMID: 32674817; PMCID: PMC7294249.

23. McGarry BE, SteelFisher GK, Grabowski DC, Barnett ML. COVID-19 Test Result Turnaround Time for Residents and Staff in US Nursing Homes. JAMA Intern Med. 2020 Oct 30:e207330. doi: 10.1001/jamainternmed.2020.7330. Epub ahead of print. PMID: 33125044; PMCID: PMC7600050.

24. Clarfield A. Mark, Orly Manor, Gabi Bin Nun, et al. Health and health care in Israel: an introduction [part of the Lancet Israel Country Series. Lancet 2017; 389:2503-13. https://doi.org/10.1016/S01406736(17) 30636-0

\section{Tables}


Table 1

- The subdivision of the LTCFs in Israel

\begin{tabular}{|llllll|}
\hline Type & Ministry of Health & \multicolumn{3}{l}{$\begin{array}{l}\text { Ministry of Social Services and } \\
\text { Social Affairs }\end{array}$} & Total \\
\cline { 2 - 5 } & $\begin{array}{l}\text { Nursing care, Skilled nursing care } \\
\text { and psycho-geriatric }\end{array}$ & Frail & Independent & $\begin{array}{l}\text { People with } \\
\text { disability }\end{array}$ & \\
\hline Facilities & 315 & 111 & 293 & 388 & 1,107 \\
\hline Residents & 33,424 & 5,200 & 41,696 & 19,726 & 100,046 \\
\hline Workers & 30,363 & 4,658 & 11,540 & 15,598 & 62,159 \\
\hline
\end{tabular}

Table 2

LTCFs COVID-19 outcomes post national screening program implementation - mortality, decreased size outbreaks and hospitalizations.

\begin{tabular}{|llll|}
\hline & $\begin{array}{l}\text { Pre national program } \\
\text { implementation } \\
\text { (Weeks = 19) }\end{array}$ & $\begin{array}{l}\text { Post national program } \\
\text { implementation } \\
\text { (Weeks = 18) }\end{array}$ & $\begin{array}{l}\text { P } \\
\text { value }\end{array}$ \\
\hline $\begin{array}{l}\text { Mortality in LTCF / Total National } \\
\text { Mortality (\%) }\end{array}$ & $(252 / 556) 45.3 \%$ & $(709 / 2337) 30.3 \%$ & 0.001 \\
\hline $\begin{array}{l}\text { Mortality (\%) over age } 75 \text { of total } \\
\text { national mortality }\end{array}$ & $(201 / 380) 52.89 \%$ & $(616 / 1487) 41.42 \%$ & $<$ \\
\hline Hospitalizations (\%) & $(2010 / 14790) 13.59 \%$ & $(4224 / 36991) 11.41 \%$ & $<$ \\
\hline $\begin{array}{l}\text { Decreased Size Outbreaks / Total } \\
\text { Outbreaks (\%) }\end{array}$ & $(48 / 235) 20.43 \%$ & $(755 / 912) 82.79 \%$ & 0.001 \\
\hline
\end{tabular}

\section{Figures}




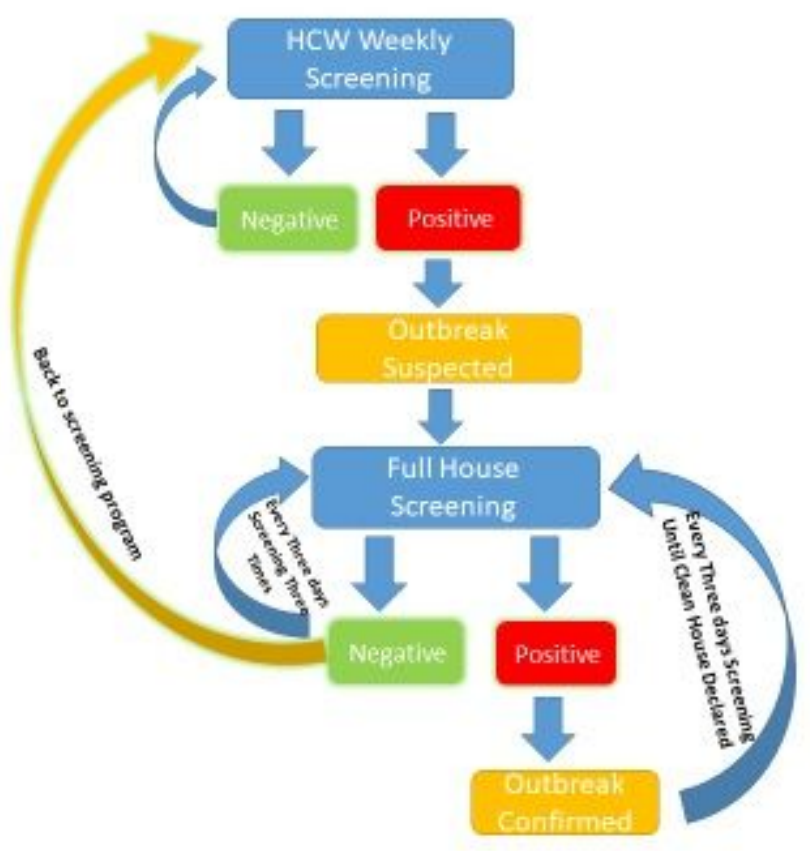

Figure 1

Flow Chart - Operational Testing Program

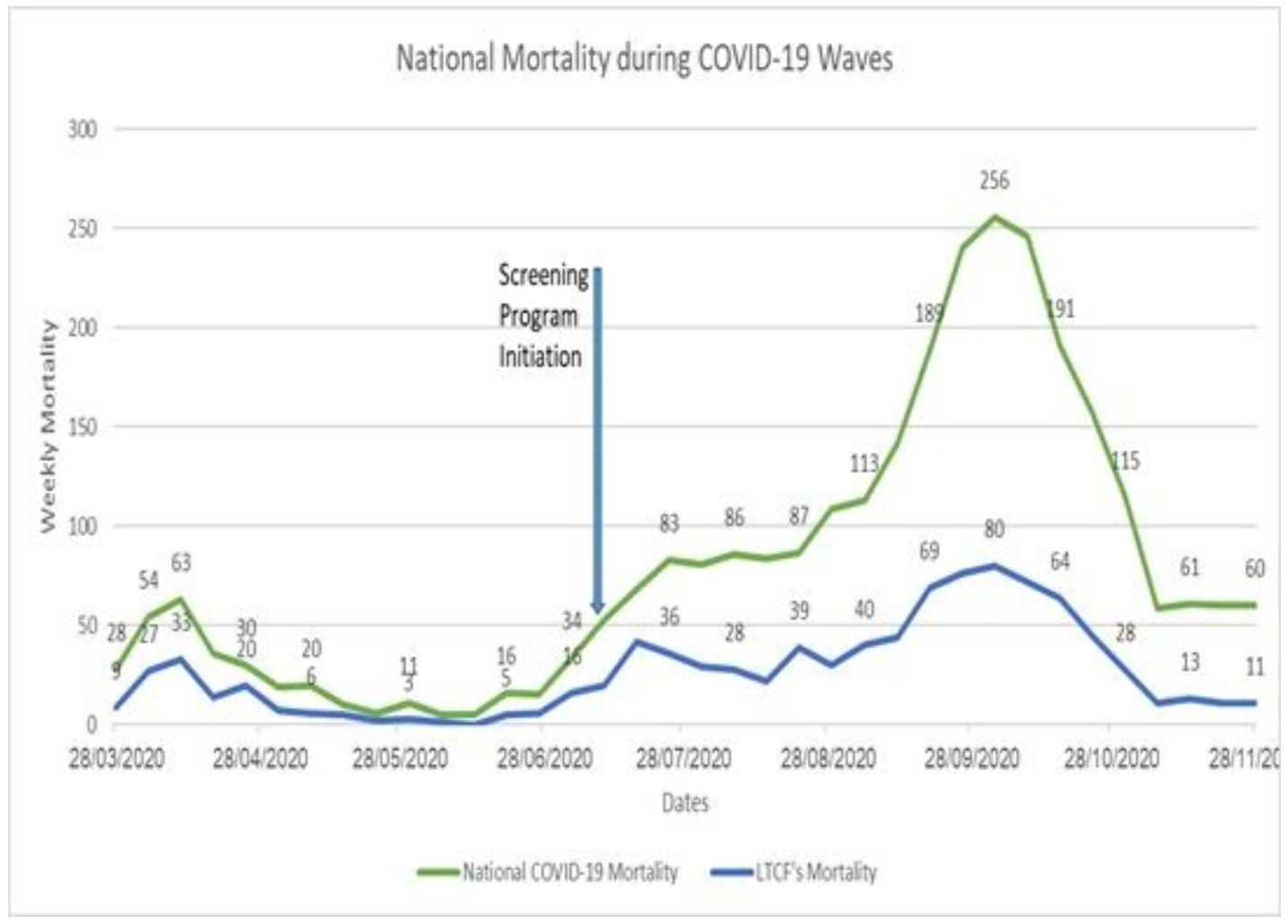

Figure 2

Weekly Israeli COVID 19 National Mortality Rate (Green) alongside LTCFs Mortality (Blue) 


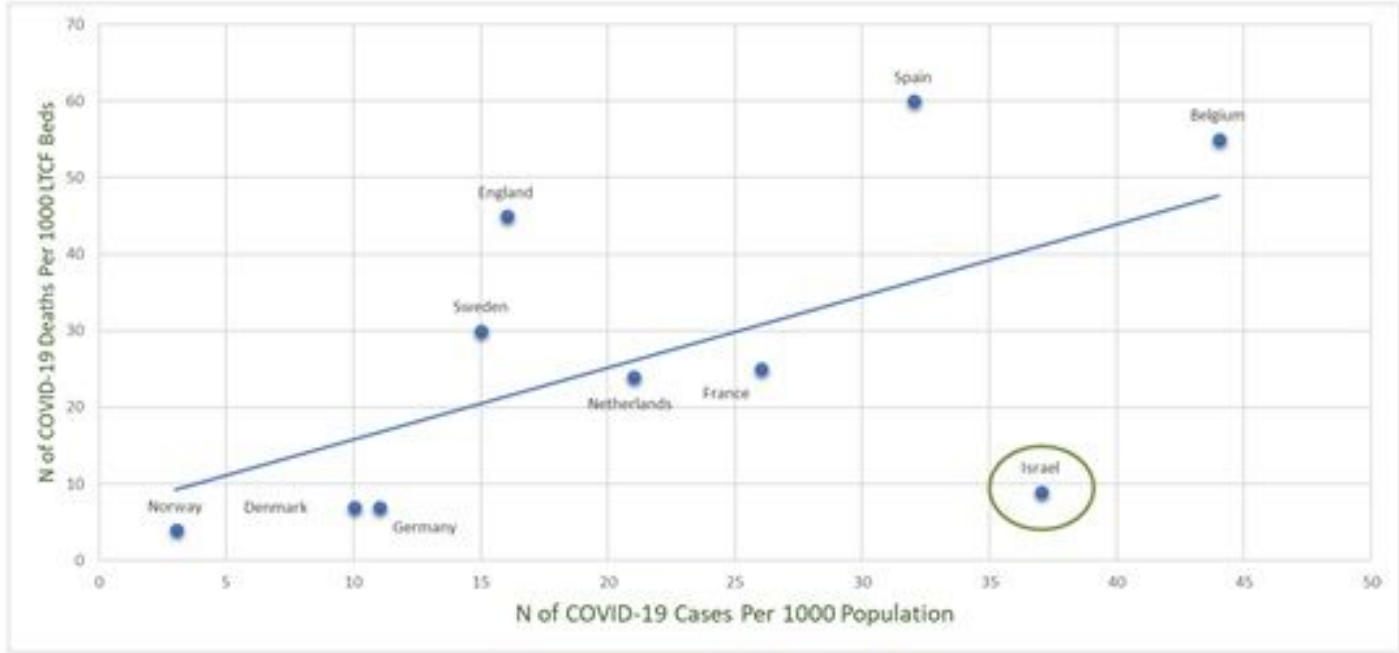

Figure 3

Covid-19 mortality per 1000 LTCF beds and COVID-19 prevalence in different European countries 